\title{
Identification of pathogenic genes and transcription factors in glaucoma
}

\author{
JIE FENG and JING XU \\ Department of Ophthalmology, The First People's Hospital of Jining, Jining, Shandong 272011, P.R. China
}

Received March 20, 2018; Accepted April 3, 2019

DOI: $10.3892 / \mathrm{mmr} .2019 .10236$

\begin{abstract}
Glaucoma is a group of eye diseases characterized by alterations in the contour of the optic nerve head, with corresponding visual field defects and progressive loss of retinal ganglion cells. The present study aimed to identify the key genes and upstream regulators in glaucoma. To screen the pathogenic genes involved in glaucoma, an integrated analysis was performed by using the microarray datasets in glaucoma derived from the Gene Expression Omnibus (GEO) database. The functional annotation and potential pathways of differentially expressed genes (DEGs) were additionally examined by Gene Ontology (GO) and Kyoto Encyclopedia of Genes and Genomes (KEGG) enrichment analyses. A glaucoma-specific transcriptional regulatory network was constructed to identify crucial transcriptional factors that target the DEGs in glaucoma. From two GEO datasets, 1,935 DEGs (951 upregulated and 984 downregulated genes) between glaucoma and normal controls were identified. GO and KEGG analyses identified that 'eye development' [false discovery rate $(\mathrm{FDR})=0.00415533$ ] and 'visual perception' (FDR=0.00713283) were significantly enriched pathways for DEGs. The expression of lipocalin 2 (LCN2), monoamine oxidase A (MAOA), hemoglobin subunit $\beta$ (HBB), paired box 6 (PAX6), fibronectin (FN1) and cAMP responsive element binding protein 1 (CREB1) were demonstrated to be involved in the pathogenesis of glaucoma. In conclusion, LCN2, MAOA, HBB, PAX6, FN1 and CREB1 may serve roles in glaucoma, regulated by PAX4, solute carrier family 22 member 1 , hepatocyte nuclear factor $4 \alpha$ and ELK1, ETS transcription factor. These data may contribute to the development of novel potential biomarkers, reveal the underlying pathogenesis and additionally identify novel therapeutic targets for glaucoma.
\end{abstract}

Correspondence to: Ms. Jing Xu, Department of Ophthalmology, The First People's Hospital of Jining, 6 Jiankang Road, Jining, Shandong 272011, P.R. China

E-mail: xujing8422@sohu.com

Key words: glaucoma, transcription factors, differentially expressed genes, integrated analysis

\section{Introduction}

Glaucoma is a widely known, multi-factorial disease, which may result in apoptosis of retinal ganglion cells. According to the World Health Organization, glaucoma is the second principal cause of blindness and the most common cause of irreversible blindness in the world $(1,2)$. In glaucoma, the anterior and posterior segments of the eye are affected, and serious damage may be detected in the trabecular meshwork (3). Oxidative stress is considered to be responsible for the molecular damage in the anterior chamber. Primary open-angle glaucoma (POAG) is the most common type of glaucoma, accounting for $60-70 \%$ all glaucoma (4). A candidate protein that may be associated with POAG is myocilin (MYOC), encoded by the MYOC gene. MYOC mutations are common in patients with POAG with high levels of intraocular pressure (IOP) $(5,6)$. Additionally, mutations in optineurin were identified in patients with POAG (7). Previous studies suggested that an abnormal expression of serine/threonine-protein kinase TBK1 is a cause of normal-tension glaucoma (8-10). Furthermore, a previous study suggested that the calcium load-activated calcium channel was involved in glaucoma and that cyclin-dependent kinase 4 inhibitor B antisense RNA 1 was upregulated in the retina of a rat model of glaucoma (11).

However, even substantial decreases in IOP are not able to prevent the development and progression of glaucoma in a number of clinical cases (12). Glaucoma-associated cell death is primarily caused by apoptosis, which is triggered by oxidative stress via mitochondrial damage, inflammation, endothelial dysregulation and dysfunction, and hypoxia (13). In general, glaucoma is not preventable; however, the vast majority of patients may maintain useful visual function for life if they have early detection and appropriate treatment (14). Therefore, for the prevention of glaucoma, emphasis must be placed on early detection, and early diagnosis and treatment.

The rapid development and application of high-throughput sequencing technology has provided a comprehensive and rapid analytical method for the study of the pathogenesis of glaucoma, and provide novel ideas for the future treatment of glaucoma (15). The present study aimed to analyze high-throughput transcriptome data from tissue samples of patients with glaucoma and a normal control group. The data was used in bioinformatics analyses to identify key transcription factors (TFs) associated with glaucoma, to examine the pathogenesis of glaucoma and provide a basis for the diagnosis of glaucoma and drug development. 


\section{Materials and methods}

Microarray expression profiling in Gene Expression Omnibus (GEO). The GEO is the largest database of high-throughput gene expression data that was developed and is maintained by the National Center for Biotechnology Information (16). The GEO was searched to obtain gene expression profiling studies of glaucoma subjects. The following key search terms were used: ['glaucoma' (Medical subject headings Terms) OR 'glaucoma' (All Fields)] AND 'Homo sapiens' (porgn) AND 'gse' (Filter). The selection criteria were as follows: i) The selected dataset must include genome-wide mRNA transcriptome data; ii) the data was obtained from the trabecular meshwork tissue samples of glaucoma and normal control trabecular meshwork tissue samples; and iii) normalized and raw datasets were considered. Following selection, two sets of GSE27276 (17) and GSE4316 (18) glaucoma mRNA data were obtained $(19,20)$.

Identification of differentially expressed genes (DEGs) in glaucoma compared with normal controls. Background correction was performed on the raw data. The normalization was performed using the Linear Models for Microarray (Limma version 3.30.13) Data package in R (21). Subsequently, two-tailed Student's t-tests were performed to calculate individual P-values. Stouffer's test was used to merge individual P-values, and multiple comparison correction was performed using the Benjamini and Hochberg method to obtain the false discovery rate (FDR) (22). Genes with FDR $<0.001$ were selected as DEGs. Finally, the DEGs in glaucoma vs. normal were identified.

Functional annotation of DEGs. Gene Ontology (GO) (23) and Kyoto Encyclopedia of Genes and Genomes (KEGG) (24) pathway enrichment analysis were performed to detect the biological functions and potential pathways associated with DEGs using GeneCoDis3 (http://genecodis.cnb.csic. es/analysis) as previously described (19). The GO functions of the DEGs were determined according to the three categories of: 'Biological process'; 'molecular functions'; and 'cellular component'. Pathway enrichment analysis was based on the KEGG database, as previously described (25).

Protein-protein interaction (PPI) network construction. In order to identify candidate genes involved in the formation of glaucoma, PPI networks of significant DEGs were constructed, according to the data from Biological General Repository for Interaction Datasets (BioGRID; http://thebiogrid.org/). Based on the existing data of protein interaction in the BioGRID database, Cytoscape (www.cytoscape.org/; (version 3.5.0) was used to search the top 100 upregulated and downregulated mRNAs. The PPI network interaction map was generated subsequent to the exclusion of genes that were not differentially expressed.

Screening for TFs of the top 20 DEGs and construction of TF regulatory network. For the top 20 DEGs, the $2 \mathrm{~kb}$ upstream promoter regions were downloaded from the University of California Santa Cruz (UCSC) Genome Browser website (genome.ucsc.edu). The TRANScription
FACtor (TRANSFAC) website match tool (gene-regulation. $\mathrm{com} /$ pub/databases.html) was subsequently used to analyze TFs capable of binding to the promoter region of the DEGs. TFs that exhibited altered expression in glaucoma with FDR $<0.001$ were selected. Position Weight Matrix scanning was used to scan the human genome sequence to obtain the protein-coding genes that were regulated by the differentially expressed TFs. Following removal of redundant information, the glaucoma-specific transcriptional regulatory network was constructed using Cytoscape software.

In silico validation of DEGs using GEO. The GEO database (GSE9944) was used to validate the expression of selected glaucoma DEGs. The expression levels of these genes were compared between the glaucoma cases and the normal group. The expression of five genes, cAMP responsive element binding protein 1 (CREB1), fibronectin 1 (FN1), keratin 19 (KRT19), lipocalin 2 (LCN2) and paired box 6 (PAX6) was detected, with the difference of expression levels presented as box-plots.

\section{Results}

Differential expression analysis of genes in glaucoma. The probes corresponding to multiple genes were removed, and the average gene expression to which multiple probes corresponded with was calculated. Finally, the intersection of 15,757 genes was obtained.

A total of two gene expression microarray datasets (GSE27276 and GSE4316) were used for the analysis. Compared with the normal controls, 1,935 DEGs in glaucoma were obtained $(\mathrm{P}<0.05)$; among these, 951 genes were upregulated and 984 genes were downregulated. The top 40 most significantly up- or downregulated genes are summarized in Table I. Among which, PAX6 (26), LCN2 (27), and MAOA (28) were downregulated and were associated with glaucoma. The DEGs were screened for clustering analysis. The heatmap produced by cluster analysis of the two sets of cDNA microarray data is presented in Fig. 1.

Functional annotation. In Fig. 2, GO enrichment demonstrated that the DEGs were significantly enriched the 'Biological processes' categories: 'eye development' $\left(\mathrm{FDR}=4.15 \times 10^{-3}\right)$; 'visual perception' (FDR $\left.=7.13 \times 10^{-3}\right)$; 'negative regulation of insulin receptor signaling pathway' ( $\left.\mathrm{FDR}=1.47 \times 10^{-2}\right)$; the 'Cellular components' categories: 'membrane' ( $\mathrm{FDR}=3.41 \times 10^{-52}$ ); 'endoplasmic reticulum' ( $\left.\mathrm{FDR}=3.76 \times 10^{-12}\right)$;

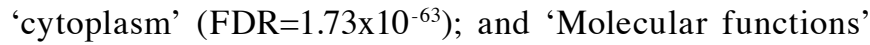
categories: 'nucleotide binding' (FDR=8.50x10 ${ }^{-16}$ ); 'hydrolase activity' ( $\left.\mathrm{FDR}=8.50 \times 10^{-16}\right)$; and 'protein binding' $\left(\mathrm{FDR}=6.15 \times 10^{-60}\right)$.

Furthermore, as presented in Fig. 3, the results of the KEGG pathway enrichment demonstrate that DEGs were enriched in 'Staphylococcus aureus infection' (FDR $=6.15 \times 10^{-60}$ ); 'Pathways in cancer' (FDR=8.66 $10^{-5}$ ); 'Systemic lupus erythematosus'; and 'Type I diabetes mellitus' ( $\left.\mathrm{FDR}=0.84 \times 10^{-3}\right)$.

PPI network construction. Following removal of the non-DEGs, the PPI network was established. The results are presented in Fig. 4. The network consisted of nodes and edges. 
Table I. Top 40 differentially expressed mRNAs.

A, Upregulated

\begin{tabular}{|c|c|c|c|c|}
\hline ID & Symbol & Combined.ES & P-value & FDR \\
\hline 3043 & HBB & 5.16018337 & 0 & 0 \\
\hline 9415 & FADS2 & 2.35332790 & $1.40 \times 10^{-9}$ & $3.68 \times 10^{-6}$ \\
\hline 56172 & ANKH & 2.33974344 & $7.72 \times 10^{-9}$ & $1.11 \times 10^{-5}$ \\
\hline 3113 & HLA-DPA1 & 2.22895504 & $1.17 \times 10^{-8}$ & $1.41 \times 10^{-5}$ \\
\hline 5538 & PPT1 & 2.27584322 & $1.72 \times 10^{-8}$ & $1.69 \times 10^{-5}$ \\
\hline 83643 & CCDC3 & 2.09336726 & $2.56 \times 10^{-8}$ & $2.32 \times 10^{-5}$ \\
\hline 81618 & ITM2C & 2.10210833 & $5.11 \times 10^{-8}$ & $3.81 \times 10^{-5}$ \\
\hline 8857 & FCGBP & 2.00251174 & $1.07 \times 10^{-7}$ & $6.25 \times 10^{-5}$ \\
\hline 4166 & CHST6 & 1.94047822 & $1.48 \times 10^{-7}$ & $7.07 \times 10^{-5}$ \\
\hline 4753 & NELL2 & 1.97462732 & $2.45 \times 10^{-7}$ & $1.07 \times 10^{-4}$ \\
\hline 8718 & TNFRSF25 & 1.80276729 & $8.11 \times 10^{-7}$ & $2.84 \times 10^{-4}$ \\
\hline 81552 & VOPP1 & 1.82118066 & $8.70 \times 10^{-7}$ & $2.91 \times 10^{-4}$ \\
\hline 2191 & FAP & 1.74786612 & $1.01 \times 10^{-6}$ & $3.09 \times 10^{-4}$ \\
\hline 187 & APLNR & 1.74800187 & $1.02 \times 10^{-6}$ & $3.09 \times 10^{-4}$ \\
\hline 51196 & PLCE1 & 1.77349072 & $1.23 \times 10^{-6}$ & $3.61 \times 10^{-4}$ \\
\hline 23676 & SMPX & 1.76299253 & $1.37 \times 10^{-6}$ & $3.78 \times 10^{-4}$ \\
\hline 51226 & COPZ2 & 1.72176939 & $1.72 \times 10^{-6}$ & $4.24 \times 10^{-4}$ \\
\hline 1290 & COL5A2 & 1.71261435 & $1.78 \times 10^{-6}$ & $4.31 \times 10^{-4}$ \\
\hline 57559 & STAMBPL1 & 1.69907540 & $1.81 \times 10^{-6}$ & $4.31 \times 10^{-4}$ \\
\hline 2331 & FMOD & 1.75952553 & $1.83 \times 10^{-6}$ & $4.31 \times 10^{-4}$ \\
\hline
\end{tabular}

B, Downregulated

\begin{tabular}{|c|c|c|c|c|}
\hline ID & Symbol & Combined.ES & P-value & FDR \\
\hline 3880 & KRT19 & -2.54377891 & $3.23 \times 10^{-10}$ & $1.70 \times 10^{-6}$ \\
\hline 116039 & OSR2 & -2.44873456 & $7.08 \times 10^{-10}$ & $2.23 \times 10^{-6}$ \\
\hline 5648 & MASP1 & -2.39197514 & $2.01 \times 10^{-9}$ & $4.52 \times 10^{-6}$ \\
\hline 79845 & RNF122 & -2.31970767 & $3.00 \times 10^{-9}$ & $5.91 \times 10^{-6}$ \\
\hline 3934 & LCN2 & -2.20821475 & $8.44 \times 10^{-9}$ & $1.11 \times 10^{-6}$ \\
\hline 57801 & HES4 & -2.22824712 & $1.65 \times 10^{-8}$ & $1.69 \times 10^{-5}$ \\
\hline 10232 & MSLN & -2.09546485 & $2.65 \times 10^{-8}$ & $2.32 \times 10^{-5}$ \\
\hline 9245 & GCNT3 & -2.11365461 & $4.07 \times 10^{-8}$ & $3.38 \times 10^{-5}$ \\
\hline 84525 & HOPX & -2.01200085 & $5.57 \times 10^{-8}$ & $3.81 \times 10^{-5}$ \\
\hline 4128 & MAOA & -2.03058309 & $7.95 \times 10^{-8}$ & $5.22 \times 10^{-5}$ \\
\hline 5080 & PAX6 & -2.09500899 & $8.92 \times 10^{-8}$ & $5.62 \times 10^{-5}$ \\
\hline 64073 & C19orf33 & -2.02417662 & $1.26 \times 10^{-7}$ & $6.86 \times 10^{-5}$ \\
\hline 3866 & KRT15 & -1.96990194 & $1.40 \times 10^{-7}$ & $6.92 \times 10^{-5}$ \\
\hline 7148 & TNXB & -1.92147744 & $1.40 \times 10^{-7}$ & $6.92 \times 10^{-5}$ \\
\hline 163732 & CITED4 & -1.94673475 & $1.94 \times 10^{-7}$ & $9.01 \times 10^{-5}$ \\
\hline 1675 & CFD & -1.97823285 & $2.68 \times 10^{-7}$ & $1.14 \times 10^{-4}$ \\
\hline 6510 & SLC1A5 & -1.95192053 & $2.77 \times 10^{-7}$ & $1.15 \times 10^{-4}$ \\
\hline 1638 & DCT & -1.84682776 & $3.22 \times 10^{-7}$ & $1.30 \times 10^{-4}$ \\
\hline 3861 & KRT14 & -1.77674842 & $7.12 \times 10^{-7}$ & $2.61 \times 10^{-4}$ \\
\hline 10053 & AP1M2 & -1.77498212 & $8.52 \times 10^{-7}$ & $2.92 \times 10^{-4}$ \\
\hline
\end{tabular}

FDR, false discovery rate.

The nodes in the network represent the proteins and the edges represent the interactions between them. There were 290 nodes and 290 edges identified. Among them, the genes with higher degrees were: FN1 (degree=92); KRT15 (degree=19); major 


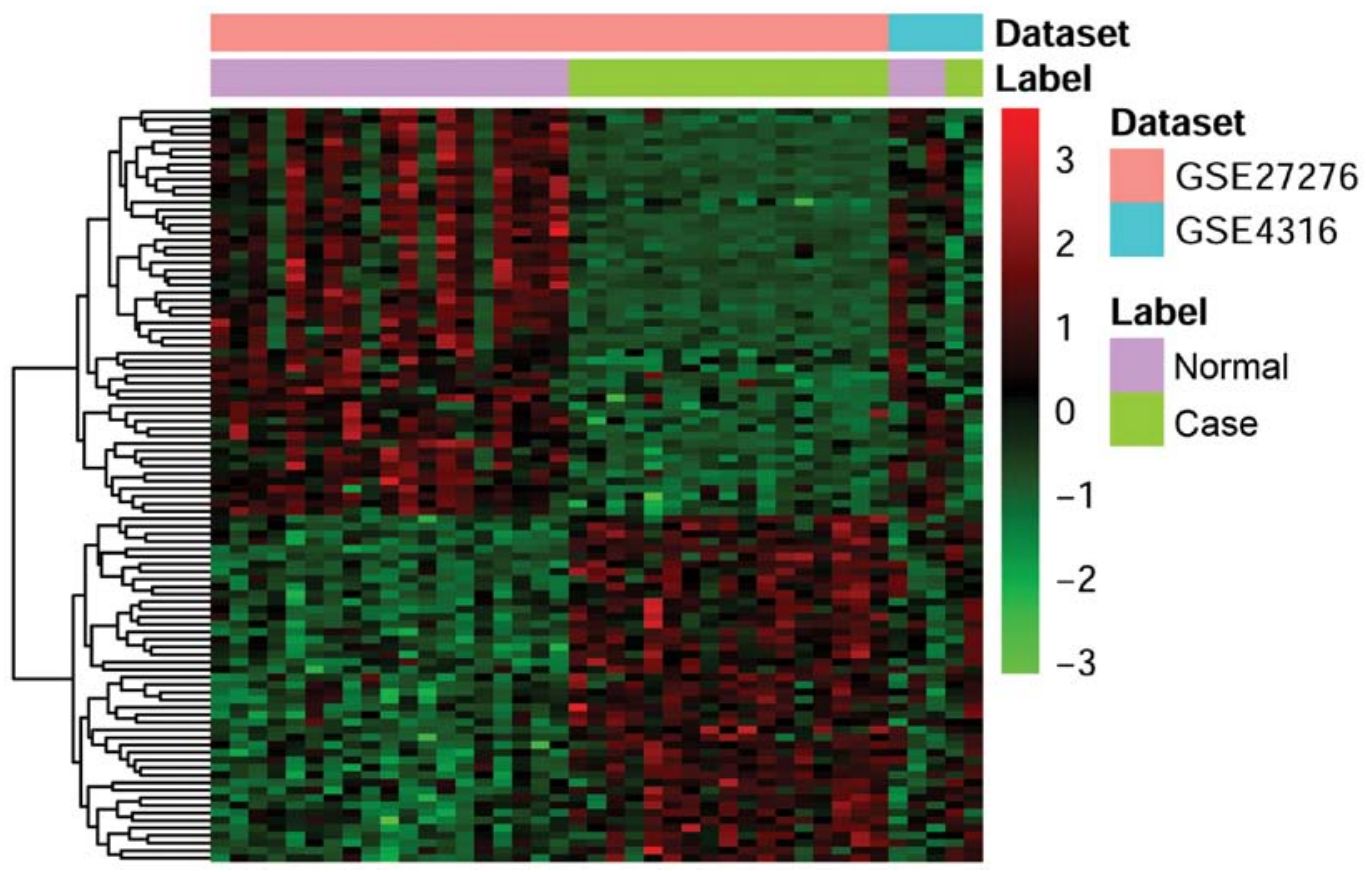

Figure 1. Heatmap of genes that were significantly up- or downregulated in glaucoma compared to normal controls. Row and column represent DEGs and tissue samples, respectively. The color scale indicated the expression of DEGs. The expression data was log transformed and standardized to zero mean and unit variance. Red and green color indicate up- and down-regulation, respectively. DEGS, differentially expressed genes.

histocompatibility complex, class II, DP $\alpha 1$ (HLA-DPA1; degree=12); CREB1 (degree=11); KRT19 (degree=9); histone cluster 2 H2B family member e (degree=9); cell division cycle associated 7 like (degree=7); PAX6 (degree=7); FOS like 2, AP-1 transcription factor subunit (degree=7); minichromosome maintenance complex component 2 (degree $=6$ ); palmitoyl-protein thioesterase 1 (degree=6); and lysine demethylase 5B (degree $=6)$. The hub proteins were FN1 (degree=92), KRT15 (degree=19) and HLA-DPA1 (degree=12).

TFs of the top 20 DEGs and TF regulation network. A total of $250 \mathrm{TF}$ binding associations were identified, including multiple binding sites of the same TF in a gene. Among these, 36 TFs were involved. In Table II, the top six TFs with the most downstream genes (the top 20 differentially expressed genes) were included: PAX4; solute carrier family 22 member 1 (1-Oct); hepatocyte nuclear factor $4 \alpha$ (HNF-4); NK2 homeobox 5 (Nkx2-5); PAX6; and ELK1, ETS transcription factor (Elk-1).

Cytoscape software was used to identify the TFs and the differentially expressed regulatory network of their target genes (Fig. 5). There were 61 nodes, including 44 transcription factors and 20 differentially expressed target genes and 132 edges identified. The eight target genes with the highest degrees were: Coiled-coil domain containing 3 (degree $=14$ ); neural tissue-specific epidermal growth factor-like repeat domain-containing protein (NELL2; degree=13); hes family bHLH transcription factor 4 (degree $=10)$; mesothelin (degree=10); integral membrane protein 2C (degree=9); fatty acid desaturase 2 (degree $=9$ ); mannan binding lectin serine peptidase 1 (degree=8); and hemoglobin subunit b $(\mathrm{HBB}$; degree=8).

Validation of DEGs in GEO GSE9944 dataset. To define the key genes with key roles in glaucoma, the GEO GSE9944 repository was searched for high throughput gene expression data and hybridization arrays, chips and microarrays. As demonstrated in Fig. 6, the genes CREB1 and FN1 were upregulated, and the genes KRT19, LCN2 and PAX6 were downregulated. These results were consistent with the integrated analysis in the GSE27276 and GSE4316 datasets.

\section{Discussion}

Glaucoma is the leading cause of irreversible blindness worldwide and there is no effective treatment at present (27). In the present study, integrated analysis was performed using data obtained from the GEO database. KEGG, GO and other biological information databases, and $\mathrm{R}$ analysis tools were used to analyze the DEGs. A total of 1,935 DEGs in glaucoma (951 genes were upregulated, 984 genes were downregulated) were obtained. Critical signaling pathways that affected the pathogenesis of glaucoma, including 'eye development' (FDR=0.00415533) and 'visual perception' (FDR=0.00713283) were identified. In addition, based on the promoter sequence of DEGs obtained from UCSC, a TF regulatory network was constructed using the match tool of the TRANSFAC website to obtain the corresponding TFs.

LCN2, encoded by $l c n 2$ gene, is a neutrophil gelatinase-associated lipocalin. The $25-\mathrm{kD} \mathrm{LCN} 2$ protein is able to bind small lipophilic substances, including bacteria-derived lipopolysaccharide and formylpeptides (28). The protein additionally functions as a modulator of inflammation. Khalyfa et al (29) suggested that LCN2 was highly upregulated in glaucoma. In the results of the present study, LCN2 was downregulated and the validation result in the GSE9944 dataset was consistent with the analysis. A previous study additionally demonstrated that the coadministration of a reversible monoamine oxidase $\mathrm{A}$ (MAOA) inhibitor with epinephrine may be useful for patients 

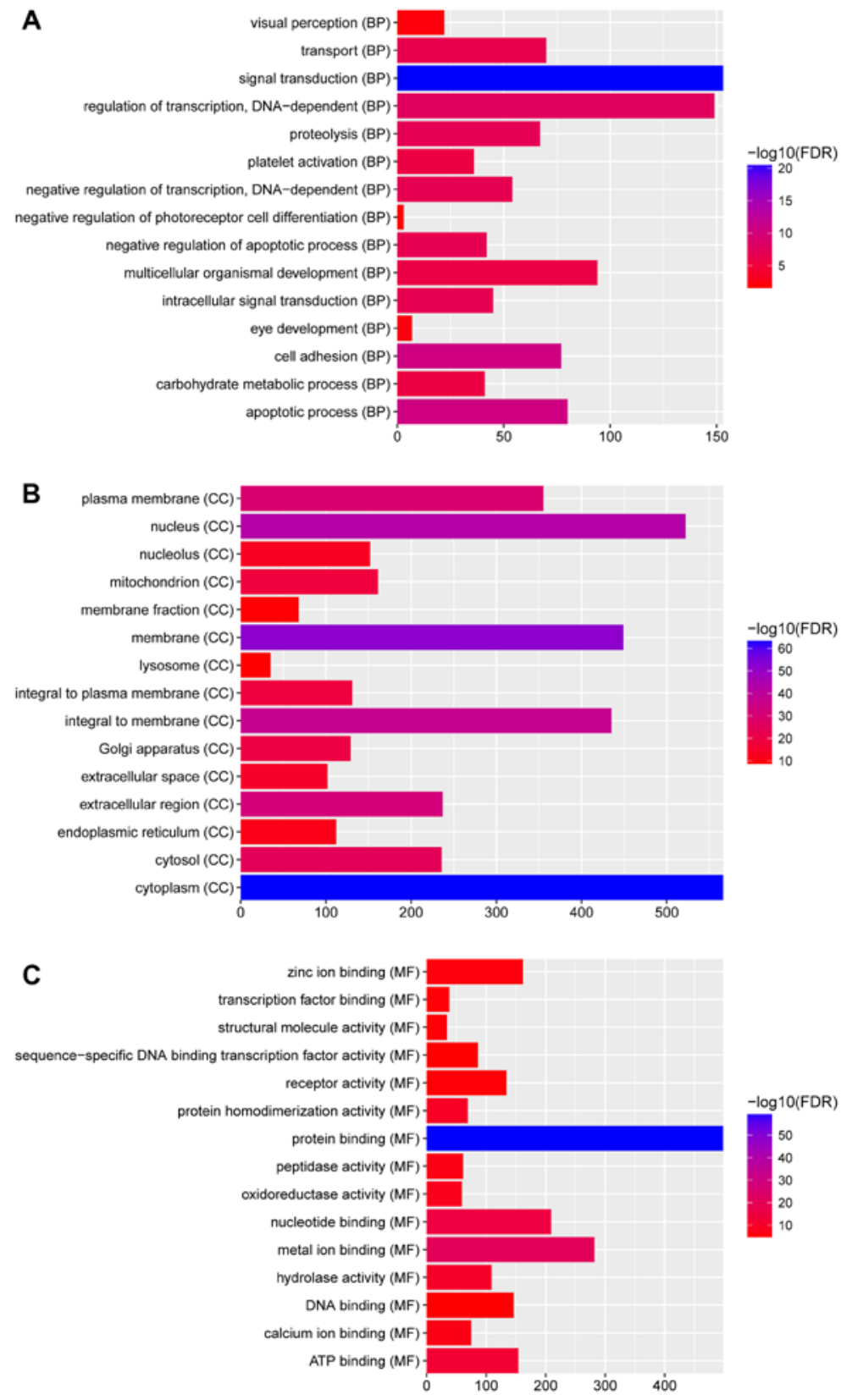

Figure 2. Gene ontology functional enrichment of differentially expressed genes (FDR<0.05). (A) BP functions. (B) CC functions. (C) MF functions. FDR, false discovery rate; BP, Biological process; CC, Cellular component; MF, Molecular functions.

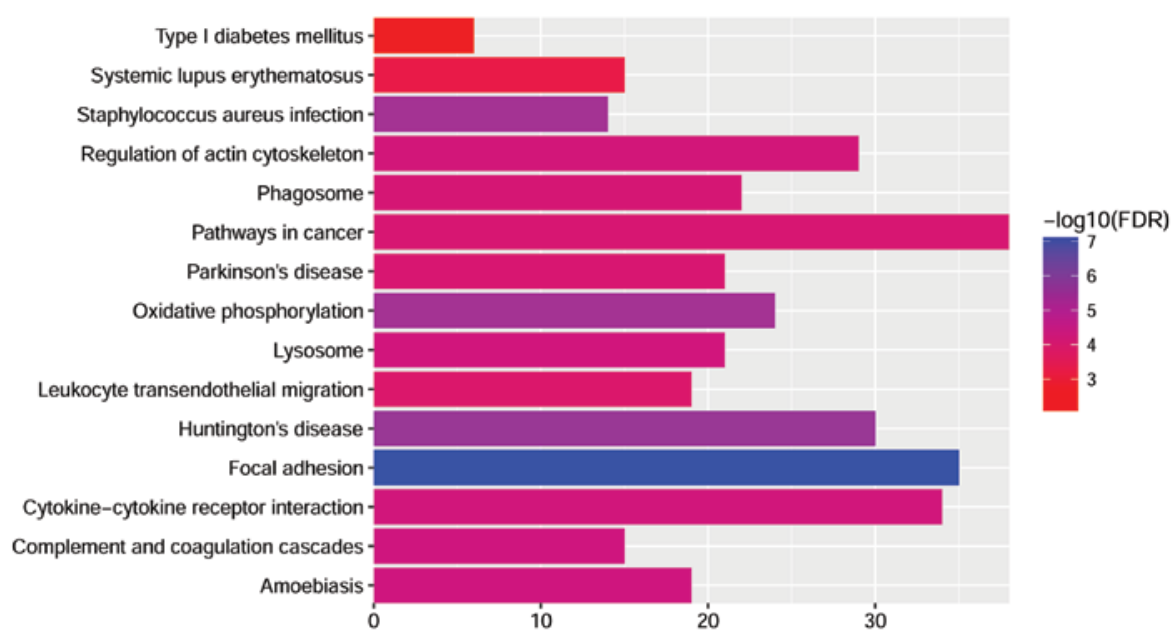

Figure 3. Kyoto Encyclopedia of Genes and Genomes analysis of differentially expressed genes in glaucoma. FDR, false discovery rate. 
Table II. Top six TFs with the highest number of downstream regulatory genes and their target genes.

Number of regulated

\begin{tabular}{|c|c|c|}
\hline TF name & genes & Regulated genes \\
\hline Pax-4 & 11 & $\begin{array}{l}\text { HES4, ITM2C, ANKH, HLA-DPA1, RNF122, LCN2, CCDC3, NELL2, MSLN, CHST6, } \\
\text { KRT19 }\end{array}$ \\
\hline $1-$ Oct & 11 & $\begin{array}{l}\text { ITM2C, MASP1, HOPX, ANKH, HLA-DPA1, OSR2, RNF122, CCDC3, HBB, NELL2, } \\
\text { MSLN }\end{array}$ \\
\hline HNF-4 & 11 & $\begin{array}{l}\text { ITM2C, MASP1, HLA-DPA1, LCN2, MAOA, HBB, FADS2, NELL2, MSLN, CHST6, } \\
\text { KRT19 }\end{array}$ \\
\hline Nkx2-5 & 9 & PPT1, HOPX, RNF122, CCDC3, FADS2, NELL2, MSLN, CHST6, KRT19 \\
\hline Pax-6 & 8 & ITM2C, MASP1, ANKH, OSR2, CCDC3, NELL2, GCNT3, KRT19 \\
\hline Elk-1 & 5 & ITM2C, MASP1, ANKH, LCN2, CCDC3, FADS2, CHST6 \\
\hline
\end{tabular}

$\mathrm{TF}$, transcription factor.

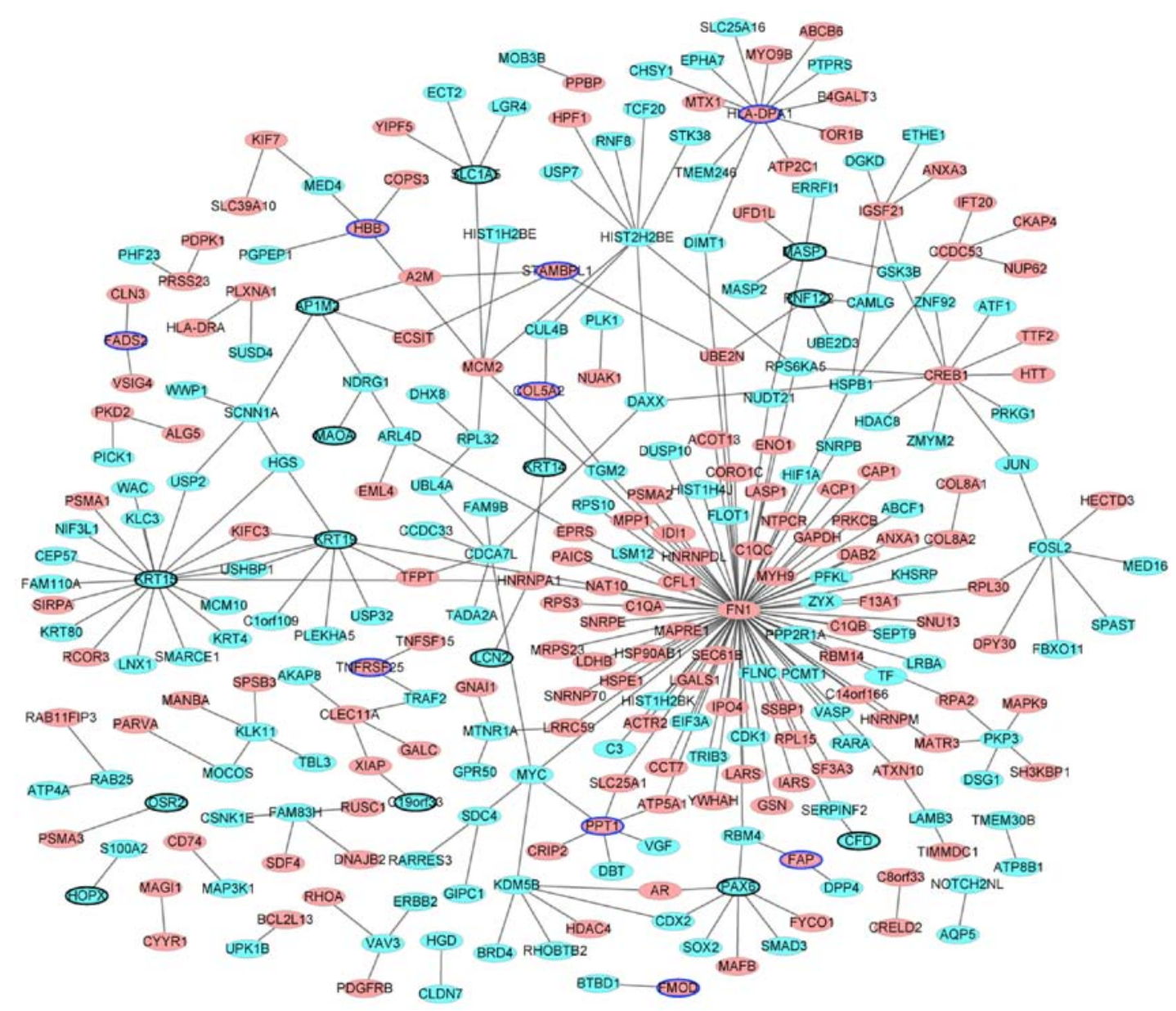

Figure 4. Protein-protein interaction network of top 100 significantly DEGs in glaucoma. The blue ellipses represent the proteins encoded by downregulated DEGs and the red ellipses represent the proteins encoded by upregulated DEGs. Among these, ellipses with black borders denote the top 10 downregulated DEGs in glaucoma. The ellipses with blue border were represented the top 10 upregulated DEGs. DEGs, differentially expressed genes.

with glaucoma (28). In the results of the present study, MAOA was downregulated.

CREB1 is a transcription regulator activated in response to harmful stress stimuli, including hypoxia and oxidative stress, and is involved in the cellular defense against these stresses (30). High IOP, optic nerve damage and visual field defects are the primary pathological features of glaucoma, with high IOP being the most common (27). CREB has been demonstrated to promote neuronal survival (31), and a previous study indicated that CREB has a neuroprotective effect against hydrogen peroxide-induced retinal ganglion cell death via 2 downstream cell survival genes, including brain-derived neurotrophic factor and apoptosis regulator Bcl-2 (32). In a study by Yasuda et al (12), an in silico pathway 


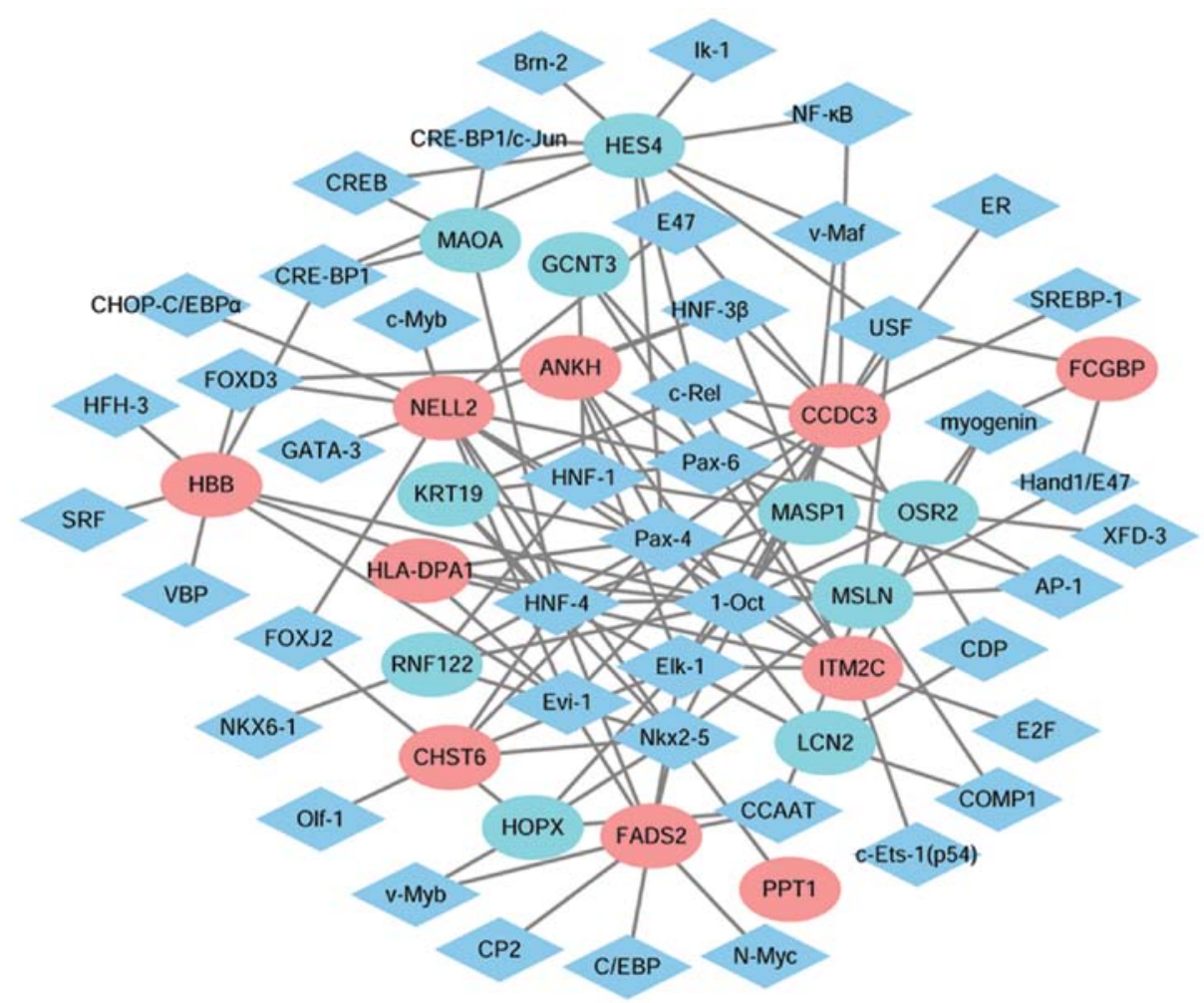

Figure 5. Glaucoma-specific transcription factors regulation network. Blue diamonds represent transcription factors, and ovals represent the top 20 differentially expressed genes. Red ovals denote upregulated genes and green ovals represent downregulated genes.
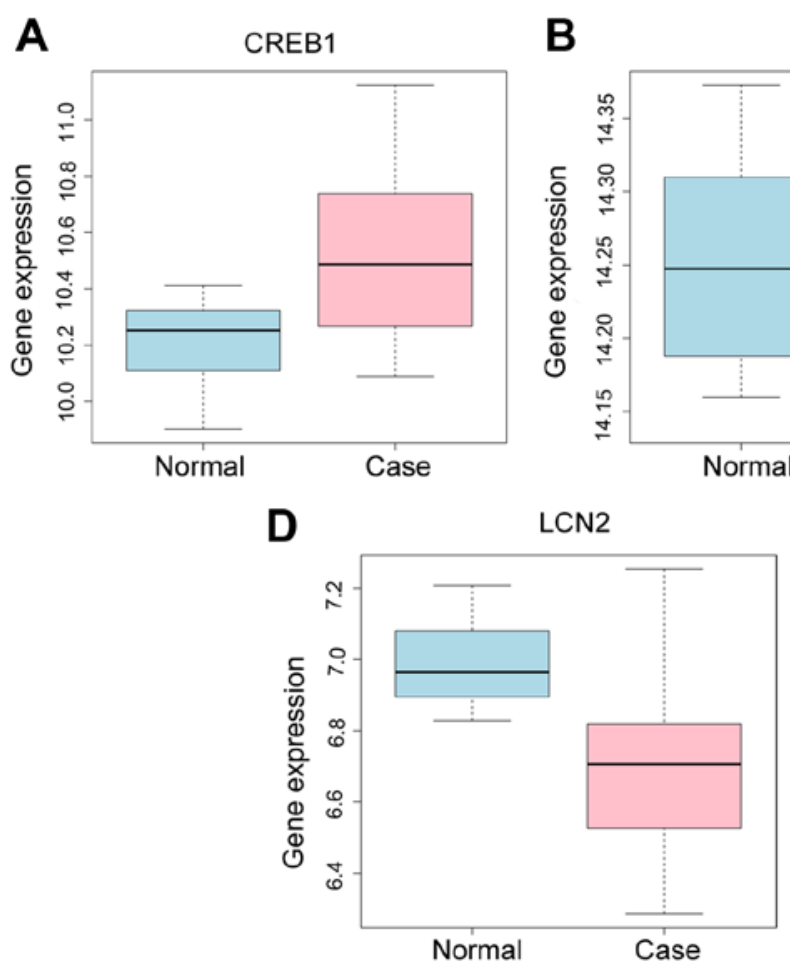

B

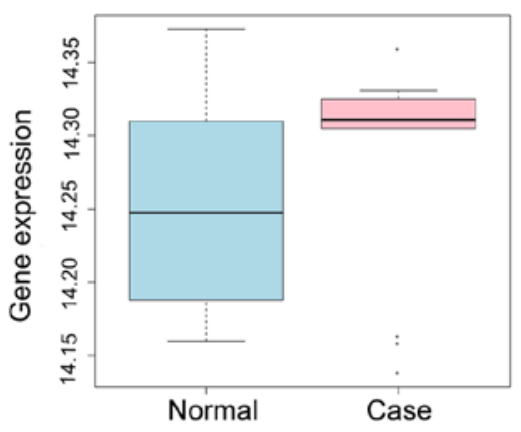

E

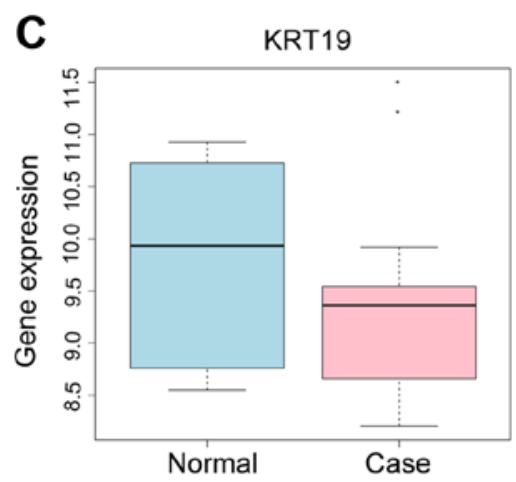

PAX6

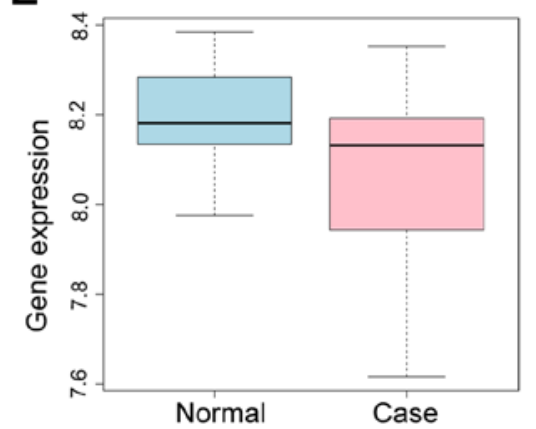

Figure 6. Validation of the expression levels of different genes in glaucoma compared with normal control based on the Gene Expression Omnibus GSE9944 database. (A) CREB1, (B) FN1, (C) KRT19, (D) LCN2 and (E) PAX6. The expression of these selected genes was consistent with the integrated analysis in the GSE27276 and GSE4316 datasets. CREB1, cAMP responsive element binding protein 1; FN1, fibronectin 1; KRT19, keratin 19; LCN2, lipocalin 2; PAX6, paired box 6.

analysis conducted in rats with axonal injury induced by transecting the right optic nerve, CREB1 was the most significant upstream regulator. In the present study, CREB1 (degree $=11$ ) was the in the top 3 genes with the highest degrees in the PPI 
network. In the KEGG analysis, CREB1 was enriched in the function of Huntington's disease.

FN1 is a glycoprotein within the extracellular matrix, which has a crucial role in cell adhesion, growth, migration and differentiation $(33,34)$. Cellular FN is secreted in a soluble form by a variety of different cell types, including fibroblasts, chondrocytes, macrophages and epithelial cells, and is later assembled into an insoluble extracellular matrix (35). FN has a multimodular structure organized into functional domains that interact with multiple binding partners, including integrins, heparin sulfate proteoglycans, collagen, glycosaminoglycans, proteoglycans, heparin, fibrin and bacteria (36). A previous study by Anshu et al (2) identified that FN1 ( $\mathrm{P}=0.0003)$ was expressed at a significantly increased level in aqueous humor samples compared with controls. In the present study, FN1 was the top protein with the highest degree in the PPI network. Additionally, in the KEGG analysis, FN1 was enriched in the function of 'Focal adhesion'.

NELL2 is a secreted glycoprotein that is predominantly expressed in neural tissues. NELL2 is additionally involved in promoting the neuronal survival required for the formation of a sexually dimorphic nucleus of the preoptic area in male rats (37). In the TF regulation network in the present study, NELL2 (degree $=13$ ) was the in top 2 genes and was regulated by PAX4, 1-Oct, HNF-4, Nkx2-5 and PAX6. Additionally, NELL2 was among the top 40 DEGs. These results indicated that NELL2 may have a key role in the pathogenesis of glaucoma.

The OMIM database was also utilized in the present study, and it was identified that PAX6 was key gene. PAX6 is a member of the paired box gene family, which encodes a transcriptional regulator involved in oculogenesis and other developmental processes. Halder et al (38) suggested that the ectopic expression of Drosophila PAX6 induces ectopic eye development. Wawersik and Maas (39) reviewed the role of PAX6 and other genes in vertebrate and fly oculogenesis; the study demonstrated that regulators of eye development are conserved across large evolutionary distances. The expression patterns and conserved functional domains suggested that Pax6 may have important roles in vertebrate eye formation. Fujimura et al (40) demonstrated that PAX6 appears to function as a pleiotropic regulator, directing the development of ocular tissues in concert with the signaling pathway and, concomitantly, regulating the expression of structural components of the eye, including shielding pigment. In the present study, PAX6 was downregulated in the patients with glaucoma. In addition, in the PPI network, PAX6 was one of the top eight genes with the highest degrees.

In conclusion, six DEGs (LCN2, MAOA, HBB, PAX6, FN1 and CREB1) were identified to be involved in the process of glaucoma. From the two GEO datasets analyzed, 1,935 DEGs (951 upregulated and 984 downregulated genes) were identified between glaucoma and normal controls. DEG validation in the GEO GSE9944 dataset was consistent with the integrated analysis of the GSE27276 and GSE4316 datasets. These results may contribute to the elucidation of novel potential biomarkers, reveal the underlying pathogenesis and identify novel therapeutic targets for the treatment of glaucoma.

\section{Acknowledgements}

Not applicable.

\section{Funding}

The present study was supported by Science and Technology Development Plan of Jining [grant no. (2016) 56-31] and Project Medical and Health Technology Development Project of Shandong (grant no. 2017WS528).

\section{Availability of data and materials}

The datasets used and/or analyzed during the current study are available from the corresponding author on reasonable request.

\section{Authors' contributions}

JF and JX conceived the study and wrote the manuscript. JX performed the data analyses. All authors have read and approved the final manuscript.

\section{Ethics approval and consent to participate}

Not applicable.

\section{Patient consent for publication}

Not applicable.

\section{Competing interests}

The authors declare that they have no competing interests.

\section{References}

1. Tham YC, Li X, Wong TY, Quigley HA, Aung T and Cheng CY: Global prevalence of glaucoma and projections of glaucoma burden through 2040: A systematic review and meta-analysis. Ophthalmology 121: 2081, 2014.

2. Anshu A, Price MO, Richardson MR, Segu ZM, Lai X, Yoder MC and Price FW Jr: Alterations in the aqueous humor proteome in patients with a glaucoma shunt device. Mol Vis 17: 1891-1900, 2011.

3. Saccà SC, Gandolfi S, Bagnis A, Manni G, Damonte G, Traverso CE and Izzotti A: From DNA damage to functional changes of the trabecular meshwork in aging and glaucoma. Ageing Res Rev 29: 26-41, 2016.

4. Kayange PC, Nkume HB, Feyi-Waboso A, Kalua K, Msukwa G and Schwering Schulze M: Presentation of primary open angle glaucoma (POAG) at lions sight first eye hospital in Blantyre, Malawi. Malawi Med J 26: 60-62, 2014.

5. Kwon YH, Fingert JH, Kuehn MH and Alward WL: Primary open-angle glaucoma. N Engl J Med 2: 1113-1124, 2009.

6. Nazir S, Mukhtar M, Shahnawaz M, Farooqi S, Fatima N Mehmood R and Sheikh N: A novel single nucleotide polymorphism in exon 3 of MYOC enhances the risk of glaucoma. PLoS One 13: e0195157, 2018

7. Huang C, Xie L, Wu Z, Cao Y, Zheng Y, Pang CP and Zhang M: Detection of mutations in MYOC, OPTN, NTF4, WDR36 and CYP1B1 in Chinese juvenile onset open-angle glaucoma using exome sequencing. Sci Rep 8: 4498, 2018.

8. Fingert JH, Robin AL, Stone JL, Roos BR, Davis LK, Scheetz TE, Bennett SR, Wassink TH, Kwon YH, Alward WL, et al: Copy number variations on chromosome 12 q14 in patients with normal tension glaucoma. Hum Mol Genet 20: 2482-2494, 2011.

9. Kawase K, Allingham RR, Meguro A, Mizuki N, Roos B, Solivan-Timpe FM, Robin AL, Ritch R and Fingert JH: Confirmation of TBK1 duplication in normal tension glaucoma. Exp Eye Res 96: 178-180, 2012.

10. Ritch R, Darbro B, Menon G, Khanna CL, Solivan-Timpe F, Roos BR, Sarfarzi M, Kawase K, Yamamoto T, Robin AL, et al: TBK1 gene duplication and normal-tension glaucoma. JAMA Ophthalmol 132: 544-548, 2014. 
11. Burdon KP, Macgregor S, Hewitt AW, Sharma S, Chidlow G, Mills RA, Danoy P, Casson R, Viswanathan AC, Liu JZ, et al: Genome-wide association study identifies susceptibility loci for open angle glaucoma at TMCO1 and CDKN2B-AS1. Nat Genet 43: 574-578, 2011.

12. Yasuda M, Tanaka Y, Omodaka K, Nishiguchi KM, Nakamura O, Tsuda S and Nakazawa T: Transcriptome profiling of the rat retina after optic nerve transection. Sci Rep 6: 28736, 2016.

13. Shi Z, Rudzinski M, Meerovitch K, Lebrun-Julien F, Birman E, Di Polo A and Saragovi HU: Alpha2-macroglobulin is a mediator of retinal ganglion cell death in glaucoma. J Biol Chem 283: 29156-29165, 2008.

14. Konstas AG, Maskaleris G, Gratsonidis S and Sardelli C: Compliance and viewpoint of glaucoma patients in greece. Eye (Lond) 14: 752-756, 2000.

15. Tajiri-Utagawa E, Hara M, Takahashi K, Watanabe M and Wakita T: Development of a rapid high-throughput method for high-resolution melting analysis for routine detection and genotyping of noroviruses. J Clin Microbiol 47: 435-440, 2009.

16. Chang WS, Wang YH, Zhu XT and Wu CJ: Genome-wide profiling of miRNA and mRNA expression in Alzheimer's disease. Med Sci Monit 23: 2721-2731, 2017.

17. Liu Y, Allingham RR, Qin X, Layfield D, Dellinger AE, Gibson J, Wheeler J, Ashley-Koch AE, Stamer WD and Hauser MA: Gene expression profile in human trabecular meshwork from patients with primary open-angle glaucoma. Invest Ophthalmol Vis Sci Sep 54: 6382-6389, 2013.

18. Liton PB, Luna C, Challa P, Epstein DL and Gonzalez P. Genome-wide expression profile of human trabecular meshwork cultured cells, nonglaucomatous and primary open angle glaucoma tissue. Mol Vis 12: 774-790, 2006.

19. Barrett T, Wilhite SE, Ledoux P, Evangelista C, Kim IF Tomashevsky M, Marshall KA, Phillippy KH, Sherman PM, Holko M, et al: NCBI GEO: Archive for functional genomics data sets-update. Nucleic Acids Rese 41 (Database Issue): D991-D995, 2013.

20. Li JJ, Wang BQ, Fei Q, Yang Y and Li D: Identification of candidate genes in osteoporosis by integrated microarray analysis. Bone Joint Res 5: 594-601, 2016.

21. Ritchie ME, Phipson B, Wu D, Hu Y, Law CW, Shi W and Smyth GK: limma powers differential expression analyses for RNA-sequencing and microarray studies. Nucleic Acids Res 43 e47, 2015 .

22. Klipper-Aurbach Y, Wasserman M, Braunspiegel-Weintrob N, Borstein D, Peleg S, Assa S, Karp M, Benjamini Y, Hochberg Y and Laron Z: Mathematical formulae for the prediction of the residual beta cell function during the first two years of disease in children and adolescents with insulin-dependent diabetes mellitus. Med Hypotheses 45: 486-490, 1995

23. Ashburner M, Ball CA, Blake JA, Botstein D, Butler $\mathrm{H}$, Cherry JM, Davis AP, Dolinski K, Dwight SS, Eppig JT, et al: Gene ontology: Tool for the unification of biology. The gene ontology consortium. Nat Genet 25: 25-29, 2000.

24. Kanehisa M and Goto S: KEGG: Kyoto encyclopedia of genes and genomes. Nucleic Acids Res 28: 27-30, 2000.

25. Wang F, Wang R, Li Q, Qu X, Hao Y, Yang J, Zhao H, Wang Q, Li G, Zhang F, et al: A transcriptome profile in hepatocellular carcinomas based on integrated analysis of microarray studies. Diagn Pathol 12: 4, 2017.
26. Dufier JL, Rozet JM, Kaplan J and Roche O: From congenital glaucoma to chronic open angle glaucoma in adulthood: A clinical and genetic continuum. Bull Acad Natl Med 197: 133-141, 2013.

27. Wang X, Huai G, Wang H, Liu Y, Qi P, Shi W, Peng J, Yang $\mathrm{H}$, Deng $\mathrm{S}$ and Wang Y: Mutual regulation of the Hippo/Wnt/LPA/TGF- $\beta$ signaling pathways and their roles in glaucoma (review). Int J Mol Med 41: 1201-1212, 2018.

28. Alpízar-Alpízar W, Laerum OD, Illemann M, Ramírez JA, Arias A, Malespín-Bendaña W, Ramírez V, Lund LR, Borregaard N and Nielsen BS: Neutrophil gelatinase-associated lipocalin (NGAL/Lcn2) is upregulated in gastric mucosa infected with Helicobacter pylori. Virchows Archiv 455: 225-233, 2009.

29. Khalyfa A, Chlon T, Qiang H, Agarwal N and Cooper NG: Microarray reveals complement components are regulated in the serum-deprived rat retinal ganglion cell line. Mol Vis 13: 293-308, 2007.

30. Maeda I, Ueda T, Koide R, Inatomi M, Fukado Y, Uchida E, Oguchi K and Yasuhara H: Ocular hypotensive effects of monoamine oxidase-a inhibitors in rabbit. Jpn J Ophthalmol 32: 211-218, 1988.

31. Ajmone-Cat MA, De Simone R, Nicolini A and Minghetti L: Effects of phosphatidylserine on $\mathrm{p} 38$ mitogen activated protein kinase, cyclic AMP responding element binding protein and nuclear factor-kappaB activation in resting and activated microglial cells. J Neurochem 84: 413-416, 2003.

32. Walton M, Woodgate AM, Muravlev A, Xu R, During MJ and Dragunow M: CREB phosphorylation promotes nerve cell survival. J Neurochem 73: 1836-1842, 1999.

33. Shao Y, Yu Y, Zhou Q, Li C, Yang L and Pei CG: Inhibition of miR-134 protects against hydrogen peroxide-induced apoptosis in retinal ganglion cells. J Mol Neurosci 56: 461-471, 2015.

34. Pankov R and Yamada KM: Fibronectin at a glance. J Cell Sci 115: 3861-3863, 2002.

35. Yi W, Xiao E, Ding R, Luo P and Yang Y: High expression of fibronectin is associated with poor prognosis, cell proliferation and malignancy via the NF- $\mathrm{NB} / \mathrm{p} 53$-apoptosis signaling pathway in colorectal cancer. Oncol Rep 36: 3145-3153, 2016

36. Jun HK, Jung YJ and Choi BK: Inflammasome activators induce fibronectin expression and release in macrophages. Cell Microbiol 19, 2017.

37. Jeong JK, Ryu BJ, Choi J, Kim DH, Choi EJ, Park JW, Park JJ and Lee BJ: NELL2 participates in formation of the sexually dimorphic nucleus of the pre-optic area in rats. J Neurochem 106: 1604-1613, 2008

38. Halder G, Callaerts P and Gehring WJ: Induction of ectopic eyes by targeted expression of the eyeless gene in Drosophila. Science 267: 1788-1792, 1995.

39. Wawersik S and Maas RL: Vertebrate eye development as modeled in Drosophila. Hum Mol Genet 9: 917-925, 2000.

40. Fujimura N, Klimova L, Antosova B, Smolikova J, Machon O and Kozmik Z: Genetic interaction between Pax6 and $\beta$-catenin in the developing retinal pigment epithelium. Dev Genes Evol 225: $121-128,2015$. 